\title{
Serum HMGB1 Levels and Its Association With Endothelial Dysfunction in Patients With Polycystic Ovary Syndrome
}

\author{
Hao-hua WANG ${ }^{1}$, Min LIN ${ }^{1}$, Guang-da XIANG ${ }^{2}$ \\ ${ }^{1}$ Department of Endocrinology, Tungwah Hospital of Sun Yat-Sen University, Dongguan, \\ P. R. China, ${ }^{2}$ Department of Endocrinology, Wuhan General Hospital of Guangzhou Command, \\ Wuhan, Hubei Province, P. R. China
}

Received December 5, 2017

Accepted May 23, 2018

On-line September 11, 2018

\section{Summary}

High-mobility group box 1 (HMGB1) is newly discovered protein, which play a crucial role in the pathogenesis of systemic inflammation. Recent studies showed that HMGB1 is one of the important pathophysiological mechanisms in the occurrence and development of atherosclerosis. The purpose of the present study was to investigate the relationship between serum HMGB1 levels and endothelial function in patients with polycystic ovary syndrome (PCOS). Eighty newly diagnosed patients with PCOS and eighty normal women of similar age were selected. Metformin treatment $(1,500 \mathrm{mg} /$ day $)$ was initiated in all patients for a period of consecutive 3 months. Serum HMGB1 levels were measured by ELISA. High resolution ultrasound was used to measure the brachial artery diameter at rest, after reactive hyperemia (flow-mediated arterial dilation, FMD) and after sublingual glyceryltrinitrate. Serum HMGB1 levels in PCOS were $24.87 \pm 14.93 \mathrm{ng} / \mathrm{ml}$, which were significantly higher than that in controls $(8.82 \pm 3.55 \mathrm{ng} / \mathrm{ml}, \mathrm{p}<0.01)$. After 3 months treatment, serum HMGB1 levels decreased significantly $(p<0.05)$. By dividing the distribution of HMGB1 levels into quartiles, serum HMGB1 levels were increased gradually with the increase of testosterone levels $(p<0.05)$, whereas the FMD levels decreased $(p<0.05)$. Multiple stepwise linear regression analysis showed that FMD (estimated coefficient $\beta=-0.69, p=0.005)$, testosterone $(\beta=0.31$, $p=0.045)$, TBARS $(\beta=0.69, p=0.012)$ and hs-CRP levels $(\beta=0.68$, $\mathrm{p}=0.001$ ) were significantly associated with HMGB1. The absolute changes in HMGB1 showed a positive correlation with the changes in testosterone $(p<0.05)$ and negative correlation with the changes in FMD $(p<0.05)$ in patients with PCOS during the course of metformin therapy. Serum HMGB1 levels are correlated with endothelial dysfunction in patients with PCOS. Our study suggests that HMGB1 may contribute to the early stage of atherosclerosis in patients with PCOS.

\section{Key words}

Endothelial function - HMGB1 • Polycystic ovary syndrome • Metformin

\section{Corresponding author}

H. Wang, Department of Endocrinology, Tungwah Hospital of Sun Yat-Sen University, Dongguan 523110, China. E-mail: hello_vanwang@sohu.com

\section{Introduction}

Polycystic ovary syndrome (PCOS) is diagnosed by the presence of hyperandrogenism, oligoanovulation, and polycystic morphology of at least one ovary (Azziz et al. 2006). PCOS has been regarded as a chronic systemic disease, and it is frequently associated with insulin resistance, hyperandrogenemia, chronic inflammation and oxidative stress (Azziz et al. 2006). It is acknowledged that PCOS is associated with increased risk for cardiovascular disease (Cibula et al. 2000, Patel et al. 2017, Gursoy et al. 2016). Endothelial dysfunction is a key early step in the development of atherosclerosis (Celermajer et al. 1992). It was well documented that endothelial dysfunction exists in subjects with PCOS (Lambert et al. 2015, Srung et al. 2014). Moreover, some studies have shown that endothelial dysfunction is partially dependent on inflammation and oxidative stress in PCOS (Pertynska- 
Marczewska et al. 2015, Hyderali and Mala 2015).

Numerous studies have focused on high-mobility group box 1 protein (HMGB1), since HMGB1 could enhance the pro-inflammatory effect of triggering receptors expressed on myeloid cells (TREM) 1, which has been recognized as a major ligand to TREM1 (Wu et al. 2012). More recently, HMGB1 is reported to have a crucial role in the pathogenesis of systemic inflammation and related with oxidative stress (Ingels et al. 2015, Kang et al. 2014). Beyond its traditional effects on the inflammatory, several studies showed that HMGB1 plays an important role in the vasculature (Hashimoto et al. 2012, Yan et al. 2009, Ito et al. 2007), and increased serum HMGB1 levels are associated with cardiovascular diseases (Hashimoto et al. 2012, Yan et al. 2009, Ito et al. 2007). However, up to now, no data are available on the relationship between HMGB1 and endothelial function in PCOS. In this study, we examined the association between HMGB1 and endothelial function in PCOS and interaction between these parameters could be the major underlying cause of cardiovascular diseases in PCOS.

\section{Subjects and Methods}

\section{Subjects}

From January 2015 to November 2017, 80 newly diagnosed patients with PCOS were studied, 80 age-matched healthy subjects served as control. The diagnosis of PCOS was based on National Institutes of Health (NIH) criteria (Azziz et al. 2006, Zawadski and Dunaif 1992). Exclusion criteria were similar to that in previous study (Pepene et al. 2011). The exclusion criteria are as follows: current or within 6 months use of oral contraceptives, anti-androgens, infertility medications, or drugs known to affect carbohydrate-lipid metabolism was declined by all subjects. Secondary causes of androgen excess such as androgen-secreting tumors, Cushing's syndrome, or medication were excluded.

Patients were administered with metformin therapy $(0.5 \mathrm{~g}$ tid) for 3 months. This study has been reviewed by an appropriate ethics committee (Thirdgrade class-A hospital) and has been conducted in accordance with the ethical standards laid down by the 1964 Declaration of Helsinki. Written informed consent was obtained from all subjects.

\section{Laboratory methods}

The procedure followed was as described previously (Wang et al. 2015). Venous blood samples were drawn after a 12 -h to 14 -h overnight fast. The serum HMGB1 levels were measured in EDTA-plasma samples by a commercially available kit (Shino-Test, IBL, Tokyo, Japan). Plasma samples were diluted $1 / 3$ and measured in duplicate and the results were averaged. The levels of triglyceride (TG), total cholesterol (TC), high-density lipoprotein cholesterol (HDL-C), low-density lipoprotein cholesterol (LDL-C), and other conventional biochemical parameters were measured on an automatic analyzer. Fasting blood glucose (FBG) was measured by glucose oxidase-color method. Sex hormone-binding globulin (SHBG, nmol/l) and high-sensitive C-reactive protein (hs-CRP, mg/l) were all measured using ELISA kits (GenWay Biotech, Inc., San Diego, USA). Total testosterone was measured by ELISA (Crystal Chem, Downers Grove, USA). The plasma lipid peroxide content was determined using thiobarbituric acid reactive substances (TBARS) as markers (Xiang et al. 2010). Insulin resistance was estimated by the homeostasis model assessment of insulin resistance (HOMA-IR) defined as fasting glucose $(\mathrm{mmol} / \mathrm{l}) *$ fasting insulin $(\mathrm{mIU} / \mathrm{l}) / 22.5$.

\section{Ultrasound study of the brachial artery}

The vascular studies of the brachial artery were performed non-invasively, as described by us previously (Wang et al. 2015). High resolution ultrasound was used to measure changes in arterial diameter in response to reactive hyperemia (with increased flow producing an endothelium-dependent stimulus to vasodilation; FMD) and to glyceryltrinitrate (GTN-induced endotheliumindependent arterial dilation $)(128 \mathrm{XP} / 10$ with a $7.0-\mathrm{MHz}$ linear array transducer; Acuson, Mountain View, CA, USA). The intra- and inter-observer variability in our laboratory for repeated measurements of artery diameter are $0.09 \pm 0.10 \mathrm{~mm}$ and $0.08 \pm 0.13 \mathrm{~mm}$, respectively. The $\mathrm{CV}$ for FMD measurements over time is 7.8-9.2\%; the power of the study to justify the selected sample size is $83.7 \%$, and the resolving power of the method tested on phantom arteries is $8.6 \mu \mathrm{m}$.

\section{Statistical methods}

SPSS 22.0 software was used to analyze all the data. Data are reported as mean $\pm \mathrm{SD}$. All individuals were classified into four quartiles according to serum HMGB1 or testosterone. The differences among the 
groups were evaluated by ANOVA test. The definition of the lower normal limit of serum HMGB1or testosterone was set at the 25th lower percentile of the HMGB1 or testosterone in patients. Univariate analysis of the effects of each potential risk factor on HMGB1 levels was done with linear regression for continuous variables, while differences in category data were assessed by the one-way analysis. Pearson's correlation was used to verify correlation between two variables. The risk factor for HMGB1 was evaluated by multiple stepwise linear regressions. Linear regression analyses were used to assess the relation between the metformin therapyinduced changes in serum HMGB1 levels and other variables. Statistical significance was set at $\mathrm{P} \leq 0.05$.

\section{Results}

Table 1 showed the clinical and biochemical characteristics of the subjects included in the study. The patients with PCOS had higher HMGB1 levels compared with the healthy subjects $(24.87 \pm 14.93 \mathrm{ng} / \mathrm{ml}$ vs. $8.82 \pm 3.55 \mathrm{ng} / \mathrm{ml}, \mathrm{p}<0.05)$ as well as lower levels of
FMD $(2.85 \pm 1.35 \%$ vs. $5.51 \pm 1.12 \%, \mathrm{p}<0.01)$. Compared with healthy women, patients with PCOS had higher BMI, HOMA-IR, TC, TG, LDL-C, TBARS, testosterone and hs-CRP levels. The patients with PCOS did not differ from the control group in age, SBP, DBP, FBG, HDL-C, SHBG, baseline vessel, baseline flow and GTN-induced dilation (Table 1).

By dividing the distribution of HMGB1 levels into quartiles (Table 2), hs-CRP levels, TBARS, testosterone, BMI, LDL-C, TC and HOMA-IR were gradually increased with increase of the serum HMGB1 levels s $(p<0.05)$. However, the FMD levels were gradually decreased with increase of the serum HMGB1 levels $(p<0.05)$. In addition, the age baseline vessel and TG showed a tendency to increase when there was a tendency toward an increase in serum HMGB1 levels ( $p>0.05$ ), while HDL-C was decreased with increase of the serum HMGB1 levels $(p>0.05)$. These four groups did not show any difference in, SBP, DBP, FBG, SHBG, baseline flow and GTN-induced dilation. Besides, patients were also divided into four quartile groups according to the testosterone levels as shown in Table 3.

Table 1. Clinical and biochemical characteristics in PCOS patients as well as in control groups $(\bar{x} \pm s)$.

\begin{tabular}{|c|c|c|c|}
\hline & \multirow{2}{*}{ Controls } & \multicolumn{2}{|c|}{ PCOS patients } \\
\hline & & Before treatment & After treatment \\
\hline Number of subjects & 80 & 80 & 80 \\
\hline Age (years) & $23.7 \pm 3.1$ & $24.5 \pm 2.6$ & $24.6 \pm 2.5$ \\
\hline$S B P(m m H g)$ & $111.7 \pm 8.7$ & $118.1 \pm 5.5$ & $112.2 \pm 5.9$ \\
\hline$D B P(m m H g)$ & $75.5 \pm 7.3$ & $76.3 \pm 6.6$ & $75.1 \pm 5.7$ \\
\hline$B M I\left(\mathrm{~kg} / \mathrm{m}^{2}\right)$ & $21.2 \pm 3.7$ & $28.5 \pm 3.5^{*}$ & $25.5 \pm 1.8 \#$ \\
\hline $\mathrm{FBG}(\mathrm{mmol} / \mathrm{l})$ & $4.06 \pm 0.85$ & $5.23 \pm 0.56$ & $4.89 \pm 0.97$ \\
\hline$T C(\mathrm{mmol} / \mathrm{l})$ & $4.23 \pm 0.33$ & $4.89 \pm 0.42 *$ & $4.12 \pm 0.33 \#$ \\
\hline$L D L-C(\mathrm{mmol} / \mathrm{l})$ & $2.69 \pm 0.23$ & $3.08 \pm 0.99 *$ & $2.89 \pm 0.11$ \\
\hline$H D L-C(\mathrm{mmol} / \mathrm{l})$ & $1.31 \pm 0.56$ & $1.28 \pm 0.16$ & $1.29 \pm 0.25$ \\
\hline$T G(\mathrm{mmol} / \mathrm{l})$ & $1.34 \pm 0.12$ & $2.21 \pm 0.64^{*}$ & $1.90 \pm 0.56$ \\
\hline$H s-C R P(m g / d l)$ & $0.04 \pm 0.06$ & $0.31 \pm 0.16^{* *}$ & $0.16 \pm 0.05 \#$ \\
\hline SHBG (nmol/l) & $38.65 \pm 13.56$ & $30.94 \pm 12.12$ & $35.56 \pm 12.23$ \\
\hline Testosterone (nmol/l) & $1.60 \pm 1.67$ & $3.88 \pm 1.55^{* *}$ & $2.11 \pm 1.33 \#$ \\
\hline$H O M A-I R$ & $1.14 \pm 1.23$ & $3.16 \pm 1.31 *$ & $1.55 \pm 1.03 \#$ \\
\hline TBARS $(\mathrm{nmol} / \mathrm{ml})$ & $1.28 \pm 0.55$ & $2.88 \pm 0.55^{* *}$ & $1.92 \pm 0.38 \#$ \\
\hline$H M G B 1(n g / m l)$ & $8.82 \pm 3.55$ & $24.87 \pm 14.93 * *$ & $13.1 \pm 6.80 \#$ \\
\hline Baseline vessel ( $\mathrm{mm})$ & $3.74 \pm 0.35$ & $3.56 \pm 0.34$ & $3.31 \pm 0.98$ \\
\hline Baseline flow $(\mathrm{ml} / \mathrm{min})$ & $86.42 \pm 18.34$ & $85.1 \pm 23.24$ & $85.12 \pm 31.33$ \\
\hline$F M D(\%)$ & $5.51 \pm 1.22$ & $2.85 \pm 1.35 * *$ & $3.45 \pm 1.31 \#$ \\
\hline GTN-induced dilation (\%) & $23.21 \pm 1.56$ & $25.11 \pm 1.78$ & $23.21 \pm 1.33$ \\
\hline
\end{tabular}

$* \mathrm{p}<0.05, * * \mathrm{p}<0.001$, compared with control, \# $\mathrm{p}<0.05$, compared with patients before treatment. 
Table 2. Clinical and biochemical characteristics of PCOS patients according to the quartiles of serum HMGB1 levels $(\bar{x} \pm s)$.

\begin{tabular}{|c|c|c|c|c|c|}
\hline \multirow{2}{*}{ Variables } & \multicolumn{4}{|c|}{ Quartiles of serum HMGB1 levels } & \multirow{2}{*}{ P-value } \\
\hline & Quartile 1 & Quartile 2 & Quartile 3 & Quartile 4 & \\
\hline Number of subjects & 20 & 20 & 20 & 20 & \\
\hline Age (years) & $21.33 \pm 4.5$ & $23.1 \pm 2.5$ & $26.2 \pm 3.5$ & $28.4 \pm 2.5$ & 0.311 \\
\hline$S B P(m m H g)$ & $115.2 \pm 3.5$ & $110.1 \pm 6.5$ & $120.5 \pm 7.1$ & $109.4 \pm 4.3$ & 0.567 \\
\hline$D B P(m m ~ H g)$ & $75.1 \pm 5.8$ & $80.5 \pm 6.1$ & $73.4 \pm 3.1$ & $77.1 \pm 3.5$ & 0.123 \\
\hline$B M I\left(\mathrm{~kg} / \mathrm{m}^{2}\right)$ & $25.3 \pm 3.1$ & $27.5 \pm 4.5$ & $28.2 \pm 3.5$ & $29.55 \pm 4.3$ & 0.021 \\
\hline$F B G(\mathrm{mmol} / \mathrm{l})$ & $4.89 \pm 0.32$ & $3.96 \pm 0.23$ & $4.55 \pm 1.25$ & $5.90 \pm 0.32$ & 0.490 \\
\hline$T C(\mathrm{mmol} / \mathrm{l})$ & $4.02 \pm 0.25$ & $4.52 \pm 0.34$ & $4.86 \pm 0.68$ & $5.23 \pm 0.67$ & 0.035 \\
\hline$L D L-C(\mathrm{mmol} / \mathrm{l})$ & $2.28 \pm 0.23$ & $2.71 \pm 0.23$ & $3.25 \pm 0.67$ & $4.11 \pm 0.34$ & 0.021 \\
\hline$H D L-C(\mathrm{mmol} / \mathrm{l})$ & $1.41 \pm 0.88$ & $1.34 \pm 0.67$ & $1.13 \pm 0.57$ & $0.99 \pm 0.31$ & 0.762 \\
\hline$T G(\mathrm{mmol} / \mathrm{l})$ & $1.78 \pm 0.55$ & $1.98 \pm 0.86$ & $2.21 \pm 0.15$ & $2.97 \pm 0.56$ & 0.137 \\
\hline$H s-C R P(m g / l)$ & $0.13 \pm 0.07$ & $0.29 \pm 0.13$ & $0.31 \pm 0.12$ & $0.45 \pm 0.19$ & 0.015 \\
\hline SHBG $(\mathrm{nmol} / \mathrm{dl})$ & $40.12 \pm 10.15$ & $28.12 \pm 13.15$ & $25.26 \pm 15.78$ & $36.23 \pm 16.33$ & 0.797 \\
\hline Testosterone (nmol/l) & $2.89 \pm 0.98$ & $3.33 \pm 189$ & $4.97 \pm 1.25$ & $5.86 \pm 1.35$ & 0.003 \\
\hline$H O M A-I R$ & $3.00 \pm 1.89$ & $3.80 \pm 1.12$ & $4.36 \pm 1.05$ & $5.23 \pm 1.98$ & 0.008 \\
\hline TBARS $(\mathrm{nmol} / \mathrm{ml})$ & $2.05 \pm 0.35$ & $2.78 \pm 0.13$ & $3.18 \pm 0.67$ & $4.12 \pm 0.89$ & 0.025 \\
\hline Baseline vessel (mm) & $2.89 \pm 0.15$ & $3.13 \pm 0.67$ & $3.88 \pm 0.56$ & $4.16 \pm 0.98$ & 0.567 \\
\hline Baseline flow ( $\mathrm{ml} / \mathrm{min})$ & $75.67 \pm 21.25$ & $90.12 \pm 21.33$ & $80.12 \pm 22.25$ & $88.12 \pm 29.25$ & 0.368 \\
\hline$F M D(\%)$ & $3.95 \pm 1.05$ & $3.05 \pm 1.15$ & $2.68 \pm 1.52$ & $2.21 \pm 1.34$ & 0.000 \\
\hline GTN-induced dilation (\%) & $31.23 \pm 2.11$ & $35.11 \pm 1.12$ & $20.67 \pm 1.23$ & $20.89 \pm 3.23$ & 0.953 \\
\hline
\end{tabular}

Table 3. Significant parameters of PCOS patients according to the quartiles of serum testosterone levels $(\bar{x} \pm s)$.

\begin{tabular}{|c|c|c|c|c|c|}
\hline \multirow{2}{*}{ Variables } & \multicolumn{4}{|c|}{ Quartiles of serum testosterone levels } & \multirow{2}{*}{ P-value } \\
\hline & Quartile 1 & Quartile 2 & Quartile 3 & Quartile 4 & \\
\hline Number of subjects & 20 & 20 & 20 & 20 & \\
\hline$T C(\mathrm{mmol} / \mathrm{l})$ & $3.96 \pm 0.12$ & $4.67 \pm 0.56$ & $4.96 \pm 0.13$ & $5.16 \pm 0.15$ & 0.025 \\
\hline$L D L-C(\mathrm{mmol} / \mathrm{l})$ & $2.06 \pm 0.12$ & $2.97 \pm 0.13$ & $3.13 \pm 0.57$ & $4.21 \pm 0.23$ & 0.032 \\
\hline$H s-C R P(m g / l)$ & $0.19 \pm 0.12$ & $0.28 \pm 0.17$ & $0.33 \pm 0.12$ & $0.40 \pm 0.11$ & 0.011 \\
\hline$H M G B 1(\mathrm{nmol} / \mathrm{l})$ & $12.65 \pm 9.96$ & $20.11 \pm 5.12$ & $27.31 \pm 13.12$ & $33.12 \pm 12.11$ & 0.001 \\
\hline$H O M A-I R$ & $3.12 \pm 1.21$ & $3.91 \pm 1.07$ & $4.52 \pm 1.12$ & $5.00 \pm 1.35$ & 0.005 \\
\hline$T B A R S(\mathrm{nmol} / \mathrm{ml})$ & $1.95 \pm 0.12$ & $2.89 \pm 0.23$ & $3.01 \pm 0.55$ & $4.28 \pm 0.57$ & 0.032 \\
\hline$F M D(\%)$ & $3.81 \pm 1.12$ & $3.08 \pm 1.23$ & $2.77 \pm 1.12$ & $2.31 \pm 1.23$ & 0.007 \\
\hline
\end{tabular}

Hs-CRP levels, TBARS, TC, LDL-C, HOMA-IR and HMGB1 gradually increased with the increase in testosterone levels (all $\mathrm{p}<0.05$ ), whereas the FMD levels gradually decreased with the increase in testosterone levels $(\mathrm{p}<0.05)$.

The changes within each group with respect to baseline variables and HMGB1 levels are presented in Table 1. BMI, HMGB1, testosterone, hs-CRP levels, TBARS, TC and HOMA-IR were statistically lower and FMD improved substantially after metformin treatment $(\mathrm{p}<0.05$ in all cases). Serum HMGB1 levels in patients before metformin treatment were $24.87 \pm 14.93 \mathrm{ng} / \mathrm{ml}$. After treatment, serum HMGB1 levels among subjects were $13.1 \pm 6.80 \mathrm{ng} / \mathrm{ml}$, which were significantly lower than those before treatment $(\mathrm{p}<0.01)$.

The FMD $(\mathrm{r}=-0.65)$, testosterone $(\mathrm{r}=0.25)$, hs-CRP levels $(\mathrm{r}=0.36)$, TBARS $(\mathrm{r}=0.56)$, TC $(\mathrm{r}=0.78)$, TG $(r=0.57)$, LDL-C $(r=0.51)$ and HOMA-IR $(r=0.33)$ in patients were significantly correlated with HMGB1 by univariate analysis. Multiple stepwise linear regression 
analysis with a age, SBP, DBP, BMI, TC, TG, LDL-C, HDL-C, testosterone, hs-CRP levels, TBARS, HOMA-IR, FMD, baseline vessel, baseline flow and GNT-induced dilation as independent variables with serum HMGB1 levels being dependent variable showed that FMD (estimated coefficient $\beta=-0.69, \mathrm{p}=0.005$ ), testosterone $(\beta=0.31, \quad p=0.045), \quad$ TBARS $(\beta=0.55, \quad p=0.012) \quad$ and hs-CRP levels $(\beta=0.37, p=0.001)$ were significantly associated with serum HMGB1 levels in patients. To reveal relations of metformin therapy-induced HMGB1 changes, linear correlation coefficients were calculated between absolute changes in serum HMGB1 levels and the parameters. The results showed a significant negative correlation between FMD and HMGB1 $(\mathrm{r}=-0.76, \mathrm{p}<0.05)$. Besides, there were significant correlations between serum HMGB1 levels and testosterone $(\mathrm{r}=0.89, \mathrm{p}<0.01)$. No significant correlations were found between changes in HMGB1 and other parameters.

\section{Discussion}

This study focused on the trigger role of HMGB1 in cardiovascular diseases, and the detailed relationship between HMGB1 and FMD in PCOS. Our results indicate the following main findings. Firstly, serum HMGB1 levels were significantly higher in PCOS than those in healthy group. Secondly, the elevated serum HMGB1 levels are positively associated with testosterone as well as negatively associated with FMD. Thirdly, serum HMGB1 decreased significantly after metformin therapy in PCOS. Changes in HMGB1 showed significant correlation with the changes in testosterone and FMD after metformin treatment. These findings suggest that decreasing extent of HMGB1 levels has beneficial effects to prevent cardiovascular diseases in PCOS.

It has been reported that chronic inflammation and oxidative stress are associated with disease progression and outcome in PCOS (Patel et al. 2017). The hs-CRP levels, produced mainly by hepatocytes, play an important role in low-grade chronic inflammation and oxidative stress (Ridker et al. 2000). Toulis et al. (2011) have shown that women with PCOS have increased levels of hs-CRP, compared with control group. Besides, Tarkun et al. (2004) found the markers of low-grade inflammation in PCOS were significantly higher than those in healthy groups. In the present study, our results showed that serum hs-CRP levels were significantly higher than controls, which is in good agreement with previous studies (Toulis et al. 2011, Tarkun et al. 2004).
Moreover, previous studies have suggested an association between PCOS and oxidative stress (PertynskaMarczewska et al. 2015, Hyderali and Mala 2015). Compared to healthy subjects, patients with POCS had significantly higher oxidative stress parameters (Pertynska-Marczewska et al. 2015). Oxidative stress increases continuously during the development of PCOS (Hyderali and Mala 2015). TBARS, an index of oxidative stress related to lipid peroxidation, were higher in PCOS compared with healthy individuals (Malin et al. 2015). Our study also confirmed previous findings that patients with PCOS have higher serum TBARS levels than healthy individuals. PCOS is associated with increased risk for cardiovascular disease (Cibula et al. 2000, Patel et al. 2017, Gursoy et al. 2016) and the impairment of FMD exists in patients with PCOS (Lambert et al. 2015, Srung et al. 2014). We also found similar results in the present study. It is acknowledged that inflammation and oxidative stress contribute to the impaired endothelial function (Xiang et al. 2010). In the present study, we demonstrated that FMD levels were negatively associated with testosterone and there was a strong correlation between testosterone and chronic inflammation, and oxidative stress in patients with PCOS. Table 3 has shown that higher testosterone level was associated with a higher incidence of chronic inflammation including CRP- concentrations, or serum TBARS levels, whereas lower FMD levels. These observations provided the foundation for the hypothesis that testosterone is able to regulate oxidative stress and inflammation through a combined mechanism, and induce endothelial dysfunction in PCOS.

HMGB1, a 25-kDa protein, which is normally located in nuclei, is passively released during cell injury and necrosis and actively secreted by some cell types via a non-canonical pathway that by passes the endoplasmic reticulum (Wu et al. 2012, Ingels et al. 2015, Kang et al. 2014). HMGB1, an inflammatory mediator, can be released from various cells under different stress conditions (Wu et al. 2012, Ingels et al. 2015, Kang et al. 2014). In vitro experiment has shown that HMGB1mediated oxidative stress and inflammation in human umbilical vein endothelial cells (Feng et al. 2013). Recent study indicated that HMGB1 could amplify oxidative stress in advanced glycoxidation end products-induced endothelial progenitor cells, a subtype of CD34-positive cells (Wu et al. 2016). In the present study, there were significant correlation between HMGB1 levels and testosterone, hs-CRP levels, TC, TG, LDL-C and 
HOMA-IR in PCOS. In addition, our study showed that testosterone, hs-CRP levels, TBARS, BMI, LDL-C, TC and HOMA-IR are increased when serum HMGB1 levels increased gradually $(\mathrm{p}<0.05)$. Multiple regression analysis showed that HMGB1 was associated with TBARS and hs-CRP levels. These results have suggested that HMGB1 may initiate and promote low grade chronic inflammation through oxidative stress in patients with PCOS. Previous studies have showed that testosterone is closely correlated with oxidative stress and may play a synergic role in the course of low grade chronic inflammation in PCOS (Beyazit et al. 2016, SavicRadojevic et al. 2015). In the present study, by dividing the distribution of testosterone levels into four quartiles, hs-CRP levels, TBARS, TC, LDL-C, HOMA-IR as well as HMGB1 were gradually increased with increase of testosterone levels $(\mathrm{p}<0.05)$. Moreover, Pearson's correlation indicated the significant relationship between testosterone levels and HMGB1. Multiple stepwise regressions showed that testosterone levels were found to be independently associated with HMGB1. In our study, patients were treated with metformin for 3 months. During treatment courses, the absolute changes in HMGB1 showed significant correlation with the changes in testosterone levels. The underlying mechanisms of increased serum HMGB1 are unclear. The increased HMGB1 levels may represent an (incomplete) mechanism that is related with oxidative stress and low grade chronic inflammation. In addition, testosterone levels are thought to regulate HMGB1 expression in our study. The alteration of testosterone levels may partially contribute to the elevated serum HMGB1 values in PCOS. Therefore, we speculate that HMGB1 play an important role in progression and outcome of PCOS, which might be related with oxidative stress and low grade chronic inflammation, and regulated by testosterone levels. Further studies are needed to assess the exact relationship between HMGB1 and PCOS.

The significance of HMGB1 in plaque formation, rupture, and thrombosis, has been identified by several studies. A clinical study of patients undergoing unstable angina and non-ST-segment elevation myocardial infarction (UA/NSTEMI) within $24 \mathrm{~h}$ of the onset of chest symptoms revealed that circulating concentration of HMGB1 on admission may be a potential and independent predictor of cardiovascular mortality in patients with UA/NSTEMI within $24 \mathrm{~h}$ of onset (Hashimoto et al. 2012). Yan et al. (2009) reported that increased serum HMGB1 level is associated with coronary artery disease in nondiabetic and type 2 diabetic patients (Yan et al. 2009). In a thrombin-induced disseminated intravascular coagulation (DIC) rat model, HMGB1 was shown to promote development of thrombosis (Ito et al. 2007). Endothelial dysfunction is an early event of atherosclerosis and plays a central role in the development and progression of cardiovascular diseases (Celermajer et al. 1992). Recently, some studies suggested that HMGB1 is associated with endothelial dysfunction in patients with type 1 diabetes and type 2 diabetes (Skrha et al. 2012). Here, we found similar results as well in PCOS. By dividing the distribution of HMGB1 levels into quartiles, we found that FMD decreased gradually as HMGB1 levels increased from quartile 1 to quartile 4 (Table 2). Besides, our data showed that the tendency of HMGB1 was associated with hs-CRP levels, TBARS, testosterone, BMI, LDL-C, TC and HOMA-IR (Table 2). In addition, age and TG are increased when serum HMGB1 levels increased gradually ( $>0.05$ ), while HDL-C was decreased with increase of the serum HMGB1 levels ( $p>0.05)$. As it is known, chronic inflammation, dyslipidemia and insulin resistance are frequently accompanied with atherosclerosis. These results can partially explain the relationship between serum HMGB1 and endothelial function in PCOS. In our study, the serum HMGB1 was negatively associated with the FMD levels through Pearson's correlation and multiple stepwise regressions, and during treatment course, the absolute changes in HMGB1 also showed significant correlation with the changes in FMD and testosterone levels. These results suggested that the higher serum HMGB1 levels may partially contribute the endothelial dysfunction in PCOS. The possible explanations for the relationship between and HMGB1 and endothelial dysfunction in PCOS are as follows: 1) Endothelial dysfunction is partially dependent on oxidative stress, low grade chronic inflammation and altered lipids profile (Xiang et al. 2010). In the present study, our results showed that serum HMGB1 levels were associated with TBARS, CRP-concentrations and altered lipids profile. These results can partially explain the relationship between serum HMGB1 and endothelial dysfunction in PCOS. 2) More studies have revealed testosterone levels to be an independent risk factor for cardiovascular diseases in PCOS (Weijenborg and Ter Kuile 2000, Dagre et al. 2006). Our results also showed that serum HMGB1 levels were positively associated with testosterone levels. 3) Imbalance in endothelial nitric oxide synthase (eNOS) activity and 
expression precedes and accelerates the development of cardiovascular diseases. There is growing evidence to suggest that HMGB1 play an important role in the inhibition of eNOS expression (Cai et al. 2017). In all, more large-scale and long-term evidence-based medical study data to further demonstrate the mechanism of the relationship between HMGB1 and endothelial dysfunction are required for a finalized conclusion. Our study suggests that targeting the HMGB1 could be a promising therapeutic strategy in early stage atherogenesis in PCOS.

This study is subject to the following limitations. Firstly, it should be better to include even larger sample size of newly PCOS in the cross-sectional study. Secondly, the patients with PCOS were not treated with anti-oxidant treatment. As a result, the changes of HMGB1 and its association with other parameters were not performed after anti-oxidant treatment. Thirdly, we did not measure the serum levels of nitrite/nitrate, which were the metabolites and the marker for production of NO. It has been well established that production of NO carries the most relevant information. In addition, other cytokines, such as irisin, chemerin and osteoprotegerin, associated with cardiovascular diseases in PCOS could not be determined, which may provide valuable information.

In conclusion, this study has shown that serum HMGB1 levels are elevated in PCOS, and are significantly associated with FMD. Thereafter, we could infer that HMGB1 plays an important role in the course of cardiovascular diseases, which is related with oxidative stress and low grade chronic inflammation and regulated by testosterone levels. Further characterization of the mechanism of HMGB1 expression, regulation, and secretion in PCOS will be required in order to develop a complete understanding of the relationship between HMGB1 and PCOS.

\section{Conflict of Interest}

There is no conflict of interest.

\section{Acknowledgements}

This work was supported by Tungwah Hospital of Sun Yat-Sen University. The authors would like to thank Professor Guangda Xiang for excellent technical support and critically revising the manuscript.

\section{References}

AZZIZ R, CARMINA E, DEWAILLY D, DIAMANTI-KANDARAKIS E, ESCOBAR-MORREALE HR, FUTTERWEIT W, JANSSEN OE, LEGRO RS, NORMAN RJ, TAYLOR AE, WITCHEL SF; ANDROGEN EXCESS SOCIETY: Positions statement: criteria for defining polycystic ovary syndrome as a predominantly hyperandrogenic syndrome: an Androgen Excess Society guideline. J Clin Endocrinol Metab 91: 4237-4245, 2006.

BEYAZIT F, YILMAZ N, BALCI O, ADAM M, YAMAN ST: Evaluation of oxidative stress in women with polycystic ovarian syndrome as represented by serum ischemia modified albumin and its correlation with testosterone and insulin resistance. Intern Med 55: 2359-2364, 2016.

CAI W, DUAN XM, LIU Y, YU J, TANG YL, LIU ZL, JIANG S, ZHANG CP, LIU JY, XU JX: Uric acid induces endothelial dysfunction by activating the HMGB1/RAGE signaling pathway. Biomed Res Int 2017: 4391920, 2017.

CELERMAJER DS, SORENSEN KE, GOOCH VM, SPIEGELHALTER DJ, MILLER OI, SULLIVAN ID: Non-invasive detection of endothelial dysfunction in children and adults at risk of atherosclerosis. Lancet 340: 1111-1115, 1992.

CIBULA D, CIFKOVA R, FANTA M, POLEDNE R, ZIVNY J, SKIBOVA J: Increased risk of non-insulin dependent diabetes mellitus, arterial hypertension and coronary artery disease in perimenopausal women with a history of the polycystic ovary syndrome. Hum Reprod 15: 785-789, 2000.

DAGRE A, LEKAKIS J, MIHAS C, PROTOGEROU A, THALASSINOU L, TRYFONOPOULOS D, DOURIDAS G, PAPAMICHAEL C, ALEVIZAKI M: Association of dehydroepiandrosterone-sulfate with endothelial function in young women with polycystic ovary syndrome. Eur J Endocrinol 154: 883-890, 2006.

FENG L, ZHU M, ZHANG M, JIA X, CHENG X, DING S, ZHU Q: Amelioration of compound, 4'-diphenylmethanebis(methyl)carbamate on high mobility group box1-mediated inflammation and oxidant stress responses in human umbilical vein endothelial cells via RAGE/ERK1/2/NF-kB pathway. Int Immunopharmacol 15: 206-216, 2013. 
GURSOY CALAN O, CALAN M, YESIL SENSES P, UNAL KOCABAS G, OZDEN E, SARI KR, KOCAR M, IMAMOGLU C, SENSES YM, BOZKAYA G, BILGIR O: Increased adipsin is associated with carotid intima media thickness and metabolic disturbances in polycystic ovary syndrome. Clin Endocrinol (Oxf) 85: 910-917, 2016.

HASHIMOTO T, ISHII J, KITAGAWA F, YAMADA S, HATTORI K, OKUMURA M, NARUSE H, MOTOYAMA S, MATSUI S, TANAKA I, IZAWA H, MARUYMA I, NOMURA M, OZAKI Y: Circulating high-mobility group box 1 and cardiovascular mortality in unstable angina and non-ST-segment elevation myocardial infarction. Atherosclerosis 221: 490-495, 2012.

HYDERALI BN, MALA K: Oxidative stress and cardiovascular complications in polycystic ovarian syndrome. Eur $J$ Obstet Gynecol Reprod Biol 191: 15-22, 2015.

INGELS C, DERESE I, WOUTERS PJ, VAN DEN BERGHE G, VANHOREBEEK I: Soluble RAGE and the RAGE ligands HMGB1 and S100A12 in critical illness: impact of glycemic control with insulin and relation with clinical outcome. Shock 43: 109-116, 2015.

ITO T, KAWAHARA K, NAKAMURA T, YAMADA S, NAKAMURA T, ABEYAMA K, HASHIGUCHI T, MARUYAMA I: High-mobility group box 1 protein promotes development of microvascular thrombosis in rats. J Thromb Haemost 5: 109-116, 2007.

KANG R, ZHANG Q, HOU W, YAN Z, CHEN R, BONAROTI J, BANSAL P, BILLIAR TR, TSUNG A, WANG Q, BARTLETT DL, WHITCOMB DC, CHANG EB, ZHU X, WANG H, LU B, TRACEY KJ, CAO L, FAN XG, LOTZE MT, TANG D: Intracellular HMGB1 inhibits inflammatory nucleosome release and limits acute pancreatitis in mice. Gastroenterology 146: 1097-1107, 2014.

LAMBERT EA, TEEDE H, SARI CI, JONA E, SHORAKAE S, WOODINGTON K, HEMMES R, EIKELIS N, STRAZNICKY NE, DE COURTEN B, DIXON JB, SCHLAICH MP, LAMBERT GW: Sympathetic activation and endothelial dysfunction in polycystic ovary syndrome are not explained by either obesity or insulin resistance. Clin Endocrinol (Oxf) 83: 812-819, 2015.

MALIN SK, KIRWAN JP, SIA CL, GONZALEZ F: Pancreatic $\beta$-cell dysfunction in polycystic ovary syndrome: role of hyperglycemia-induced nuclear factor- $\mathrm{KB}$ activation and systemic inflammation. Am J Physiol Endocrinol Metab 308: E770-E777, 2015.

PATEL SS, TRUONG U, KING M, FERLAND A, MOREAU KL, DOROSZ J, HOKANSON JE, WANG H, KINNEY GL, MAAHS DM, ECKEL RH, NADEAU KJ, CREE-GREEN M: Obese adolescents with polycystic ovarian syndrome have elevated cardiovascular disease risk markers. Vasc Med 22: 85-95, 2017.

PEPENE CE, ILIE IR, MARIAN I, DUNCEA I: Circulating osteoprotegerin and soluble receptor activator of nuclear factor $\mathrm{KB}$ ligand in polycystic ovary syndrome: relationships to insulin resistance and endothelial dysfunction. Eur J Endocrinol 164: 61-68, 2011.

PERTYNSKA-MARCZEWSKA M, DIAMANTI-KANDARAKIS E, ZHANG J, MERHI Z: Advanced glycation end products: a link between metabolic and endothelial dysfunction in polycystic ovary syndrome? Metabolism 64: 1564-1573, 2015.

RIDKER PM, HENNEKENS CH, BURING JE, RIFAI N: C-reactive protein and other markers of inflammation in the prediction of cardiovascular disease in women. $N$ Engl J Med 342: 836-843, 2000.

SAVIC-RADOJEVIC A, BOZIC ANTIC I, CORIC V, BJEKIC-MACUT J, RADIC T, ZARKOVIC M, DJUKIC T, PLJESA-ERCEGOVAC M, PANIDIS D, KATSIKIS I, SIMIC T, MACUT D: Effect of hyperglycemia and hyperinsulinemia on glutathione peroxidase activity in non-obese women with polycystic ovary syndrome. Hormones (Athens) 14: 101-108, 2015.

SKRHA J JR, KALOUSOVA M, SVARCOVA J, MURAVSKA A, KVASNICKA J, LANDOVA L, ZIMA T, SKRHA J: Relationship of soluble RAGE and RAGE ligands HMGB1 and EN-RAGE to endothelial dysfunction in type 1 and type 2 diabetes mellitus. Exp Clin Endocrinol Diabetes 120: 277-281, 2012.

SRUNG VS, JONES H, PUGH CJ, AZIZ NF, DAOUSI C, KEMP GJ, GREEN DJ, CABLE NT, CUTHBERTSON DJ: Endothelial dysfunction in hyperandrogenic polycystic ovary syndrome is not explained by either obesity or ectopic fat deposition. Clin Sci (Lond) 126: 67-74, 2014. 
TARKUN I, ARSLAN BC, CANTURK Z, TUREMEN E, SAHIN T, DUMAN C: Endothelial dysfunction in young women with polycystic ovary syndrome: relationship with insulin resistance and low-grade chronic inflammation. J Clin Endocrinol Metab 89: 5592-5596, 2004.

TOULIS KA, GOULIS DG, MINTZIORI G, KINTIRAKI E, EUKARPIDIS E, MOURATOGLOU SA, PAVLAKI A, STERGIANOS S, POULASOUCHIDOU $\mathrm{M}$, TZELLOS TG, MAKEDOS A, CHOURDAKIS M, TARLATZIS BC: Meta-analysis of cardiovascular disease risk markers in women with polycystic ovary syndrome. Hum Reprod Update 17: 741-760, 2011.

WANG HH, ZHANG XW, CHEN WK, HUANG QX, CHEN QQ: Relationship between serum irisin levels and urinary albumin excretion in patients with type 2 diabetes. J Diabetes Complications 29: 384-389, 2015.

WEIJENBORG PT, TER KUILE MM: The effect of a group program on women with the Mayer-Rokitansky-KüsterHauser syndrome. BJOG 107: 365-368, 2000.

WU J, LI J, SALCEDO R, MIVECHI NF, TRINCHIERI G, HORUZSKO A: The proinflammatory myeloid cell receptor TREM-1 controls Kupffer cell activation and development of hepatocellular carcinoma. Cancer Res 72: 3977-3986, 2012.

WU H, LI R, WEI ZH, ZHANG XL, CHEN JZ, DAI Q, XIE J, XU B: Diabetes-induced oxidative stress in endothelial progenitor cells may be sustained by a positive feedback loop involving high mobility group box-1. Oxid Med Cell Longev 194: 3918, 2016.

XIANG GD, PU JH, SUN HL, ZHAO LS: Alpha-lipoic acid improves endothelial dysfunction in patients with subclinical hypothyroidism. Exp Clin Endocrinol Diabetes 118: 625-629, 2010.

YAN XX, LU L, PENG WH, WANG LJ, ZHANG Q, ZHANG RY, CHEN QJ, SHEN WF: Increased serum HMGB1 level is associated with coronary artery disease in nondiabetic and type 2 diabetic patients. Atherosclerosis 205: 544-548, 2009.

ZAWADSKI JK, DUNAIF A: Diagnostic criteria for polycystic ovary syndrome: towards a rational approach. In: Polycystic Ovary Syndrome. DUNAIF A, GIVENS JR, HASELTINE FP, MERRIAM GR (eds), Blackwell Scientific, Boston, 1992, pp 377-384. 